\title{
A diabetes profile of the eight districts in the public health sector, Eastern Cape Province, South Africa
}

\author{
N Sahadew, BTech (Podiatry), MMedSc; V S Singaram, BMedSc, MMedSc, PhD \\ Clinical and Professional Practice, School of Clinical Medicine, College of Health Sciences, Nelson R Mandela School of Medicine, \\ University of KwaZulu-Natal, Durban, South Africa
}

Corresponding author: N Sahadew (nsahadew@gmail.com)

\begin{abstract}
Background. The International Diabetes Federation (IDF) recently reported that there are 1.8 million South Africans with diabetes, and estimates an additional undiagnosed population of $69 \%$ of the total number of diabetics. The African continent is expected to see the highest increase in diabetes globally by 2045 . Healthcare measures to manage this surge in diabetes and its related complications should be tailored to Africa's unique challenges; however, the epidemiolocal data essential for policy development are lacking. Bridging the data gap will guide funding distribution and the creation of evidence-based initiatives for diabetes.

Objectives. To investigate the frequency, age proportion and distribution of new patients diagnosed with diabetes in the public healthcare sector of Eastern Cape (EC) Province, South Africa (SA).

Methods. All data collected to date were obtained from the EC District Health Information System. According to the information collected from the Department of Health, diabetes-related data collection fields were implemented in 2013, which resulted in this 4-year study. Additional open-source data on population estimates, mortality and medical aid coverage were provided by Statistics SA.

Results. Of the eight districts in the province, O R Tambo was recorded as having the highest average proportion of new patients diagnosed with diabetes. A positive correlation was found between the calculated incidence of disease and the diabetes mortality rate.

Conclusions. The study showed an annual growth in the incidence of diabetes in the EC since 2014, and highlights the issue of an increasing burden of diabetes in the rural population. This increase is consonant with predictions by authoritative bodies on the growing burden of diabetes in Africa. The pattern of distribution highlights the deprived district of O R Tambo contradicting the well-known link between diabetes and urbanisation.
\end{abstract}

S Afr Med J 2019;109(12):957-962. https://doi.org/10.7196/SAMJ.2019.v109i12.13972

The increasing worldwide prevalence of diabetes has placed the spotlight on non-communicable diseases (NCDs) and lifestyle diseases. ${ }^{[1]}$ While this exposure is a step in the right direction, funding is disproportionate to the burden of disease, especially in sub-Saharan Africa. ${ }^{[1,2]}$ Less than $0.2 \%$ of the USA's healthcare funding for developing countries is allocated to NCDs. ${ }^{[1]}$

South Africa (SA) has made a formal commitment to reduce the burden of NCDs in the form of the National Development Plan. The implementation of a sugar tax, an expanding NCD data collection and an increase in the 2018 budget allocation for NCDs are evidence of the progress towards this goal. ${ }^{[3]}$ These steps are reassuring, but more intense effort is needed to manage the predicted surge in patients with diabetes and its complications. ${ }^{[2,4]}$

In Africa, diabetes is responsible for high rates of disability and premature mortality, and is estimated to cost at least 3.5 billion international dollars. ${ }^{[5]}$ Prevalence predictions by the International Diabetes Federation (IDF) are often surpassed, with the literature estimating the mortality rate of NCDs to exceed that of infectious diseases by $2035{ }^{[6]}$ Considering the preventive nature of type 2 diabetes and its complications, policy regarding screening and treatment protocols is essential. Quality data collection and vigorous analysis are required to improve the design of these control mechanisms to compensate for the challenges of poor infrastructure, access to healthcare, large patient loads and competing burdens of HIV and tuberculosis (TB)..$^{[2,7]}$

HIV and TB are healthcare priorities for the National Department of Health (DoH). However, large urban areas and major cities face an increasing burden of disease and mortality owing to diabetes. ${ }^{[3,4]}$
Recent studies have highlighted a high burden of disease in some deprived districts of KwaZulu-Natal (KZN) Province. ${ }^{[7]}$ Diabetes was recently identified as the major natural cause of death in the district of Nelson Mandela Bay in Eastern Cape (EC) Province. ${ }^{[4]}$ However, there is very limited research detailing the diabetes burden in this high-risk district. Our study aims to create a diabetes profile of all EC districts. Bridging the current data gap will allow for more evidencebased initiatives, policy development and tailored clinical guidelines to manage diabetes within the urban African framework.

\section{Methods}

\section{Context}

The EC, home to the area formerly known as Transkei, has been identified as one of the poorest provinces in SA. This can be attributed partly to high levels of poverty in the former homelands. ${ }^{[8]}$ The province is divided into two metropolitan municipalities and six district municipalities, with $63 \%$ of the area scoring a rank of 1 (most deprived) in the SA Index of Multiple Deprivation (SAIMD) study. ${ }^{[4]}$

The District Health Information System (DHIS) is tasked with the storage of primary data collected by allocated information officers and healthcare workers from each district. Submitted data are formatted within specific data collection fields, as prescribed by the provincial DoH.

\section{Study design}

This descriptive study involves the collection, review and analysis of data from the DHIS, which is the primary data source among the 257 districts of the nine provinces of the DoH. 
A retrospective audit of the DHIS was conducted to determine the burden of diabetes in the EC.

\section{Study sample}

All diabetes-related data collected during 2013 - 2016 (4 years) were requested.

\section{Data collection}

The following diabetes-related data collection fields have been implemented in the EC for collection by the DHIS:

- 'Diabetes patient - new.' (The number of patients newly diagnosed with diabetes.)

- 'Diabetes patient -18 years and older.' (The total number of patients diagnosed with diabetes (DoH register), who are $\geq 18$ years of age.)

- 'Diabetes patient - younger than 18 years.' (The total number of patients diagnosed with diabetes (DoH register), who are $<18$ years of age.)

Population and mortality estimates were requested from Statistics SA. Medical scheme coverage was retrieved from the 2016 District Health Barometer, published by the National Health Trust. ${ }^{[4]}$ The medical scheme coverage is the percentage of sampled population who subscribe to private medical aid schemes and are assumed to have access to medical care in the private sector.

\section{Data analysis}

Data were initially analysed using Excel 2016 (Microsoft, USA). Time series and crosssectional analyses were made possible using pivot tables, and provided insight into data trends and incidence rates. Graphical representation was developed using the software program think-cell version 6 (think-cell Inc, USA).

\section{Ethical approval}

Ethical approval was obtained from the University of KZN (ref. no. HSS/1835/017D) and the EC DoH (ref. no. EC_201711_010). Data were requested via the National Health Research Database through a dedicated online portal. No patient identifiers were used; hence, anonymity was guaranteed.

\section{Results}

The results display graphical representations of the data collected regarding newly diagnosed diabetic patients in the EC, and aim to enhance visualisation and understanding regarding the evolving status of diabetes in the province.

Fig. 1 depicts a categorical representation of the number of patients diagnosed with diabetes in the EC by year.
As illustrated in Fig. 1, 10335 patients in the EC public health sector were diagnosed with diabetes in 2013. This number decreased by $30 \%$ the following year. According to the data, the number of patients recorded by the DHIS as newly diagnosed diabetics has increased consistently since 2014 by an average of $32.03 \%$.

Fig. 2 depicts the 4-year distribution of new patients throughout the EC. The largest proportion (27\%) is seen in the coastal district of O R Tambo and the smallest proportion $(4.62 \%)$ in the district of Joe Gqabi.

The incidence of diabetes was determined using the number of new patients (Fig. 1) and population estimates, as provided by Statistics SA. The results are depicted in Fig. 3. It shows the 2013 - 2016 incidence (4-year rate) of diabetes in all eight districts of the EC. According to the most recent data, 134 in every 10000 people in the EC were diagnosed with diabetes in 2016.

Medical scheme utilisation rates were retrieved for the two major metropolitan

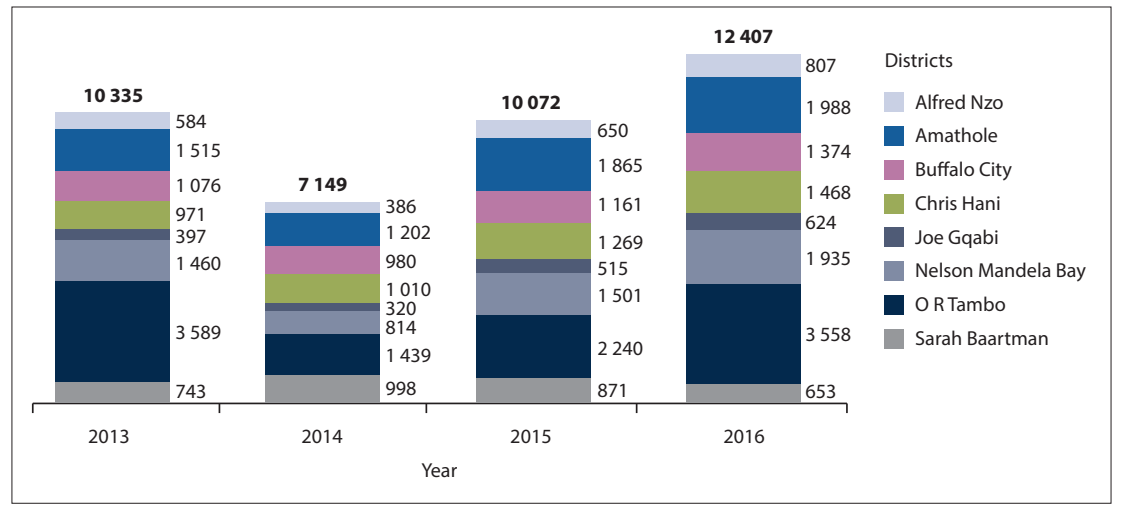

Fig. 1. Number of new patients in the districts of the Eastern Cape public health sector.

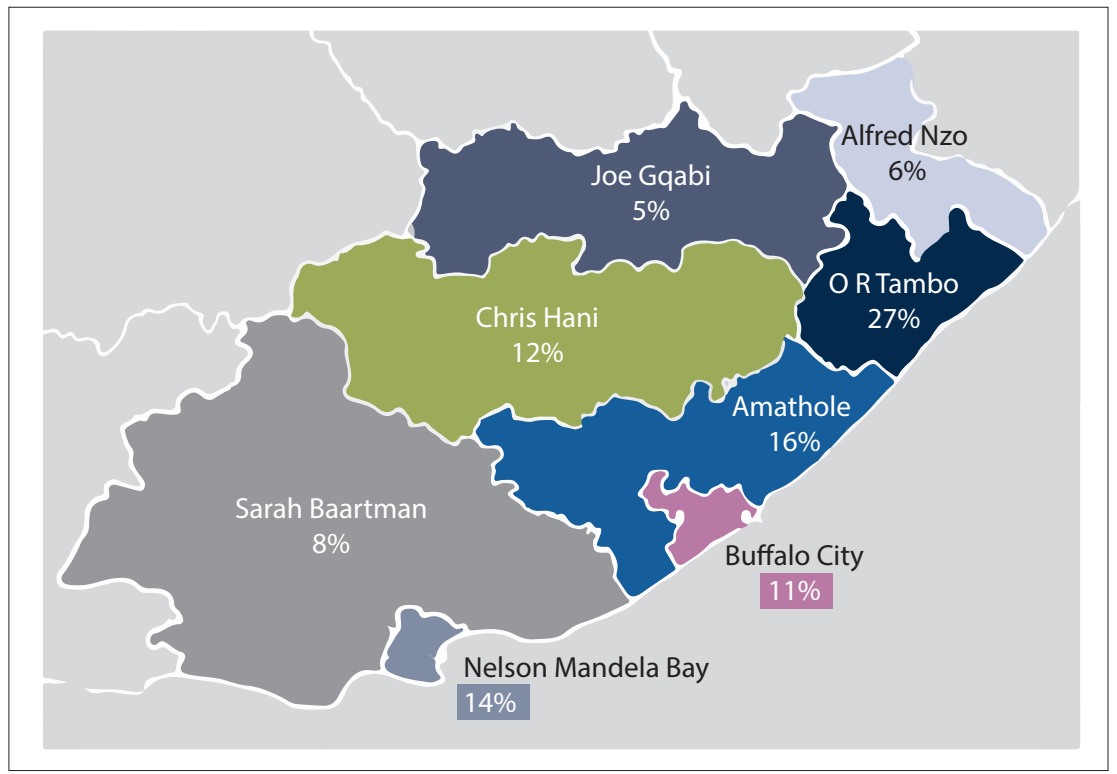

Fig. 2. Map showing the average distribution of new patients in the districts of the Eastern Cape public health sector. districts of the EC from Statistics SA (general household survey, 2016) (Fig. 4). These were used to reduce the population to exclude those with access to private medical care, resulting in a more precise estimate of the incidence of diabetes for 2016. The updated incidence rates indicate an increase of $>20 \%$

Fig. 5 illustrates the proportionate age distribution for 2016. For each district, the number of new patients $\geq 18$ years of age far outweighs that of patients $<18$ years old.

\section{Discussion}

Outcome 1: Reduced incidence of diabetes in 2014

Data detailing the number of new patients are illustrated in Fig. 1 and were used to infer the incidence of diabetes (Fig. 3). Both graphs portray a similar trend, showing a reduction in 2014, followed by a steady annual increase thereafter. This was primarily due to a common data set being used as the numerator for both calculations. across all districts. 


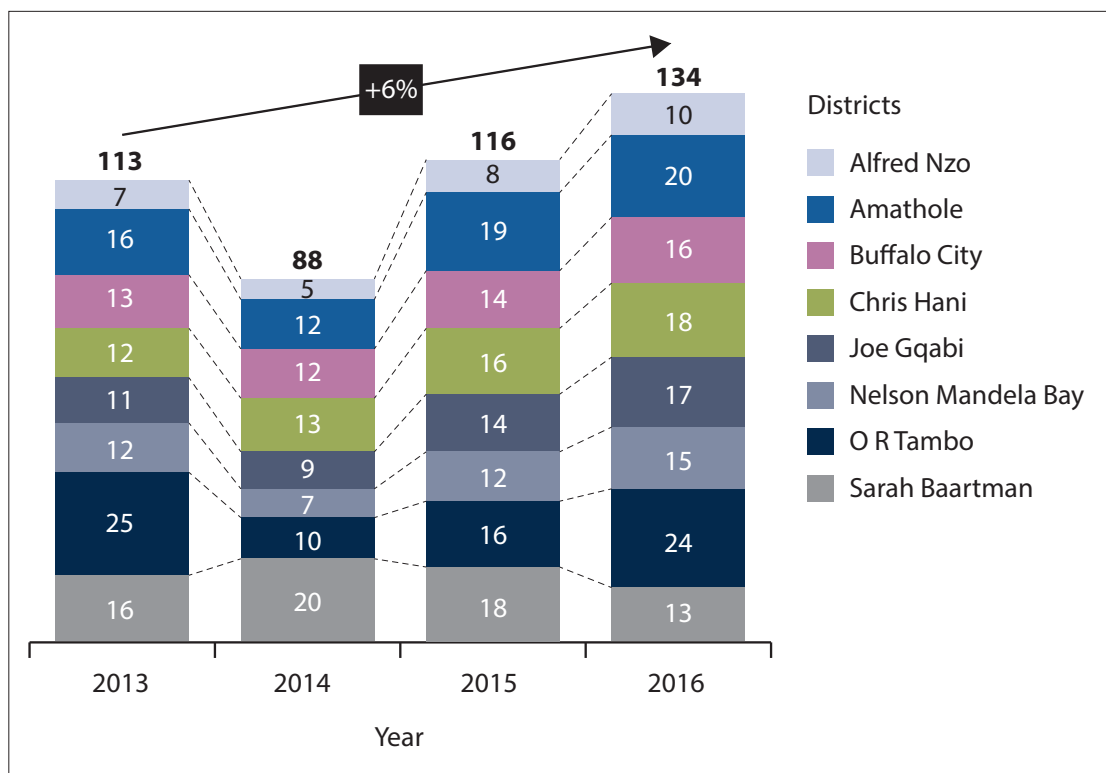

Fig. 3. Crude incidence of diabetes in the districts of the Eastern Cape public health sector (per 10000 people).

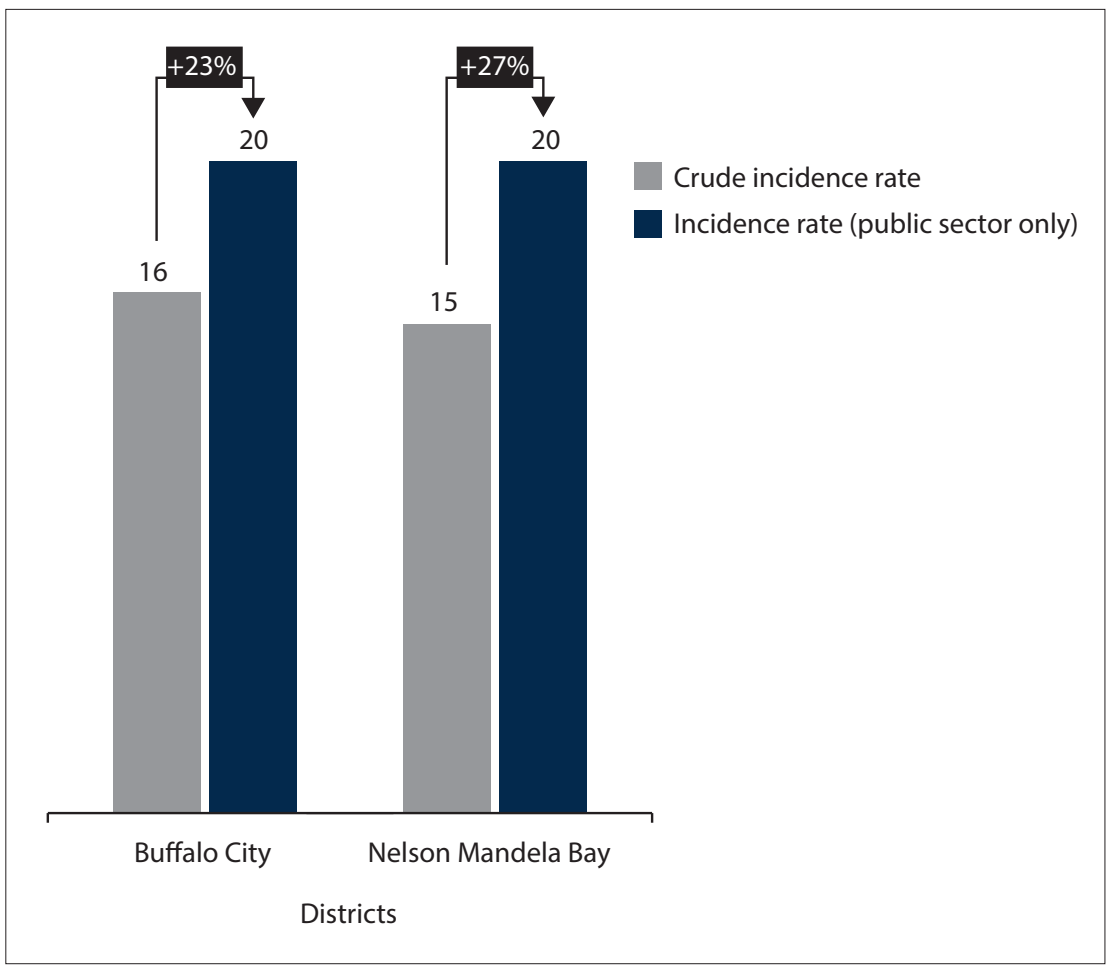

Fig. 4. Incidence of diabetes in two districts of the Eastern Cape public health sector (per 10000 people) (2016).

The reduction in 2014 is incongruent with results found in other provinces. Studies sampling 2014 data from the KZN DHIS show an increase in the number of patients and a corresponding incidence in diabetes. ${ }^{[7,9]}$

The IDF serves as a global authority on the growing epidemic of diabetes. To develop their biennial atlas, the IDF collects comprehensive data from as many countries as possible. According to data published in the IDF Atlas 6th and 7th editions (2013 and 2015, respectively), ${ }^{[10,11]}$ SA experienced a reduction in the prevalence of diabetes in 2014. When comparing editions 6 and 7 of the IDF Atlas, the diabetes prevalence is seen to have decreased from 8.2 to 7.0. This brief improvement in the burden of diabetes is echoed in data collected from the EC. The challenges of a large undiagnosed population and insufficient data on the continent were acknowledged, even though no explicit reason for the reduction was stated in either publication.

A plausible motive for this documented reprieve could point to an increase in the quality or quantity of epidemiological data, resulting in more accurate prevalence estimations. Changes in data collection fields and data interpretation are common in the DHIS, and progress is made annually to improve the quality of information they provide. ${ }^{[7,12]}$

Outcome 2: Annual increase in new patients and incidence of diabetes

Since 2014, a steady increase in the number of new patients and corresponding incidence in diabetes has been observed. This trend is similar to the global increase of the disease. ${ }^{[1]}$

The increased prevalence of diabetes is estimated to be more pronounced in subSaharan Africa due to the unique challenges in the region. ${ }^{[1,2]}$ The high degree of inequality, urbanisation and infectious diseases compounds the burden of diabetes, increasing morbidity and mortality, and straining the already stressed public health sector. ${ }^{[5]}$ In $2015,79 \%$ of diabetes-related deaths in Africa occurred in patients $<60$ years of age. This had a crippling effect on the workforce and economy, especially considering the debilitating complications of the disease that have an impact on the quality of life. ${ }^{[5]}$

Studies evaluating the cost of diabetes have estimated the minimal annual cost for an African country as 3.5 billion international dollars, exceeded only by the indirect costs, which are more difficult to quantify. ${ }^{[5]}$

In addition to having the highest projected increase in diabetic patients, sub-Saharan Africa has the lowest rate of diagnosis. This suggests the potential for a large population of undiagnosed patients and limited capacity for early care or preventive medicine.

It is imperative to review and design more targeted initiatives to improve the management of the emerging diabetes epidemic to reduce the abovementioned socioeconomic consequences.

Outcome 3: DHIS in the Eastern Cape - insufficient depth in diabetesrelated data collection

The principal aim of the DHIS has been to improve the performance of health services provided to SA communities. The system is intended to be used by stakeholders at various levels to ensure the constant evaluation of desired outcomes. These outcomes include analysis of current service delivery, prediction of future service needs, support of decentralised decision-making, informing healthcare policy and tracking of predetermined targets. ${ }^{[12]}$

Since its implementation in 1999, studies assessing or utilising the DHIS have identified multiple challenges. ${ }^{[12,13]}$ Shared 


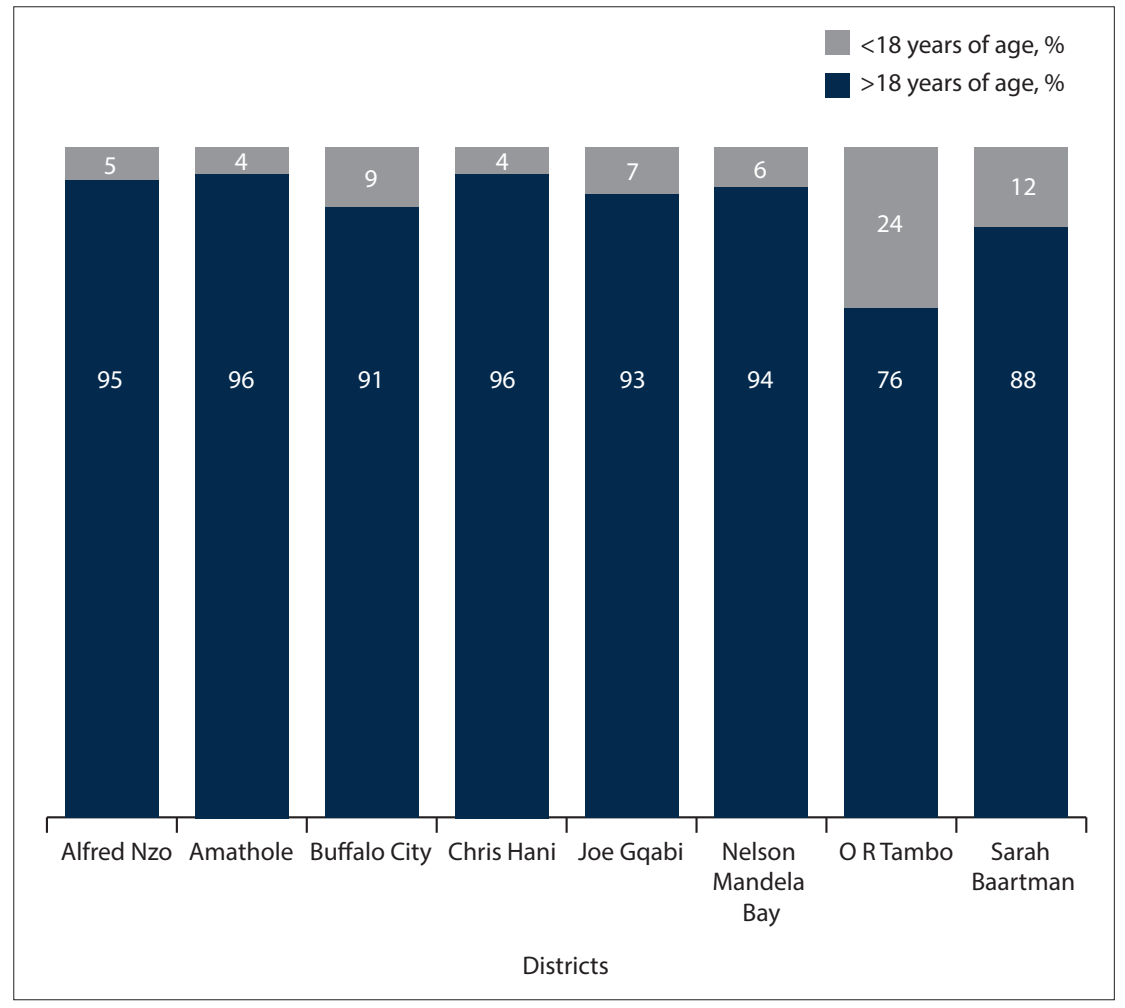

Fig. 5. Proportional distribution of diabetes by age categories in the districts of the Eastern Cape public health sector (2016).

challenges highlighted by this study include missing data fields, no explanation of data collection errors, inconsistent format of collection and unreliable data quality. ${ }^{[12]}$

The initial study period intended for analysis by the researcher (NS) was 2012 2016 (5 years). After consultation with the EC DoH, it was established that diabetesrelated data were only collected since 2013, as directed by the provincial DoH. On close inspection, three relevant data elements were identified, the total number of new patients and their distinction by age ('younger than 18 years' and ' 18 years and older'). As a result, limited insight can be gained into the diabetes burden in the EC.

The indicators used for data collection by the DHIS vary between provinces. The coastal province of $\mathrm{KZN}$, for example, collects supplementary data such as the number of patients on the register and those initiated on treatment. ${ }^{[7,9]}$ To gain further insight into the effects of the disease, the KZN DHIS records the number of diabetesrelated amputation and defaulting patients. These additional collection fields can be used to determine patient compliance and prevalence of disabling complications to direct the design of healthcare initiatives. ${ }^{[7]}$

In the EC, there is sparse recording of diabetes-related complications, which are usually limited to data from a single hospital. ${ }^{[13]}$ High mortality rates associated with hyperglycaemic emergencies, substandard medical care and poor screening have been reported in the EC public health sector. The DHIS, however, remains an underutilised tool in the province.

One study reported high rates of diabetesrelated amputations in the EC. ${ }^{[14]}$ This data collection field has been approved by the national DHIS, as implemented in KZN since 2013. ${ }^{[9]}$ However, this data indicator remains isolated to $\mathrm{KZN}$, despite the previously substantiated need in the EC. ${ }^{[14]}$

Implementing the additional nationally accepted indicators in the EC, the DHIS would allow for more extensive exploration of the distribution and burden of diabetes, thus guiding resource allocation, clinical protocol and practitioner ratios.

In addition to the standardisation of data collection fields across all provinces, the following strategies could be used to improve the quality of data collected by the DHIS and to overcome challenges identified by our study:

- More specialised training to ensure stakeholders have an improved understanding of the importance of goodquality data and the use of each data element being collected. ${ }^{[12,13,15]}$

- Review of current trouble-shooting techniques and algorithms for more accurate detection and correction of data inconsistencies. ${ }^{[12,13,15]}$
- Creation of a feedback loop between data collectors, clinic managers and policy makers to ensure the constant evaluation of data quality and DHIS efficiency. This process will also ensure that each stakeholder understands their contribution to reaching the final desired outcome. ${ }^{[12,13,15]}$

- Review of specific data collection fields with input from end users to improve the robustness of DHIS-based research and increase DHIS utilisation. In the case of diabetes-related data, the outcomes of this research could have been improved with a data collection field recording the number of diabetic patients on the $\mathrm{DoH}$ register over a 10 -year period, where each patient is only counted once. This would enable the calculation of a crude prevalence estimation. Crude prevalence is an epidemiological calculation that could allow us to gauge more accurately the national and provincial burden of disease.

\section{Outcome 4: Distribution does not follow the typical pattern of urbanisation}

With regard to the proportional distribution of newly diagnosed diabetics (Fig. 2), the district of $\mathrm{O} R$ Tambo presented with the largest proportion (27\%). This is an unusual result, as the district has been allocated a score of 1 in the SAIMD study. ${ }^{[4]}$ This study categorised districts into quintiles by calculating their individual levels and types of deprivation $(1=$ most deprived, $5=$ least deprived) - the more deprived the district, the more rural the area. ${ }^{[4]}$

There is a well-documented association between diabetes and urbanisation. ${ }^{[7,16]}$ The established risk factors for diabetes associated with urbanisation include: lack of physical activity, diet high in salt content, obesity and substance abuse. ${ }^{[16]}$ Furthermore, highly urbanised areas (cities) have larger populations and better access to specialist healthcare. ${ }^{[17]}$ Rural residents often travel to their nearest city to access tertiary medical care, which is then recorded.

This relationship would suggest that the more highly urbanised districts of Buffalo City and Nelson Mandela Bay (NMB) would have the highest proportion of patients newly diagnosed with diabetes. In 2016, diabetes was responsible for the greatest number of naturally occurring deaths in NMB ${ }^{[4]}$ This finding supports the theory of under-reporting in the DHIS, especially considering the lateness of relevant data collection in the EC. 


\section{Outcome 5: Increasing prevalence of diabetes in urban and rural settings}

As discussed in outcome 4, the pattern of distribution seen in this study does not follow the typical pattern of urbanisation. Previous studies indicated a strong correlation between prevalence of diabetes and degree of urbanisation, suggesting that the more urbanised the district, the higher the prevalence of diabetes. ${ }^{[7]}$

The findings from this sample regarding distribution indicate that rural populations present with their own unique challenges relevant to diabetes and insulin resistance, even though these populations do not face the well-known lifestyle changes that predispose urban dwellers to the disease.

One study found the diabetes prevalence to be $\sim 17 \%$ higher in the sampled rural population than in the corresponding urban sample. ${ }^{[17]}$ However, the quality of data from rural areas has been known to be unreliable due to small sample sizes, poor recording systems and limited protocol adherence. Possible reasons for high rates of diabetes in rural areas include: limited access to patient education, poverty, lack of medical resources and insufficient specialist care. ${ }^{[17]}$

\section{Outcome 6: Increased burden of disease when the private sector is excluded}

The incidence of diabetes is seen to increase from the original calculations of crude incidence (Fig. 4).

This finding is unsurprising, as the only change in the new calculation is a reduced denominator resulting in an increased quotient or crude incidence rate. The most recent private medical scheme data were obtained for the major cities in the EC. These data were used to exclude private medical scheme users from the original denominator of the total district population. Therefore, a more accurate representation of the incidence of diabetes in the public sector was achieved.

Together with revised data collection fields, updated medical aid utilisation rates would more closely reflect the current burden of disease and direct appropriate intervention.

Additional value may be gained by comparing updated results of the burden of disease in the public sector with those calculated for the private sector in the same province. Variables such as quality of care, socioeconomic background and treatment methods can be assessed for their impact on the burden of disease and prognosis of diabetes patients.

\section{Outcome 7: Data indicate the under-reporting of type 2 diabetes in the public sector}

Type 2 diabetes is typically seen in patients $>30$ years of age, while type 1 diabetes presents in early adolescence. More exceptions to this unofficial rule have been noted in the recent literature. However, $>90 \%$ of diabetic patients remain categorised as type $2{ }^{[18]}$ Fig. 5 displays the proportional distribution of diabetic cases categorised by patient age ( $<18$ and $\geq 18$ years). The districts of $O \mathrm{R}$ Tambo and Sarah Baartman have a larger than usual population of diabetic patients $<18$ years of age. Considering their young age, it is highly likely that these patients were diagnosed as type 1 diabetics.

It is unusual that the proportion of type 1 diabetics exceeds $10 \%$, which suggests possible under-reporting of diabetes cases ( $\geq 18$ years) in the districts of the EC, and further highlights the reality of a large undiagnosed population.

The insidious nature of diabetes and the high-stress environment of urbanised African countries have been credited with the creation of a large undiagnosed population. ${ }^{[18]}$ Studies reported up to $85 \%$ of type 2 diabetes patients remaining undiagnosed, with at least $20 \%$ presenting with complications at the time of diagnosis. ${ }^{[18]}$

\section{Study limitations}

The quality of the findings could be improved with an increased number of relevant data collection fields, increased duration of the data collection period and fewer data inconsistencies, such as missing entry fields in the data sample.

\section{Conclusions}

Diabetes care within a complex system such as the public sector requires a systemic approach. ${ }^{[18]}$ The many elements include competency of staff, an uninterrupted supply of medication, functional equipment and efficient data recording. ${ }^{[18]}$ The challenges faced by the EC are not limited to those identified in this research. The lack of sufficient infrastructure, traditional cultural perceptions of obesity and interactions between infectious diseases contribute to the uniqueness of the diabetes crisis in Africa. ${ }^{[1,7,14]}$

Good-quality data collected through an efficient health information system are essential for the management of healthcare services. ${ }^{[12]}$ The DHIS was intended to be used by policy makers, healthcare managers and workers to gain insight into their quality of service, predict the needs of the community and monitor their predetermined targets. ${ }^{[12]}$ Among the many challenges faced by the DHIS, this research has highlighted the need for review of an essential and consistent data set. ${ }^{[19]}$

According to our findings, the following initiatives could improve the recording, analysis and status of the burden of diabetes in the EC:

- restructured data collection fields

- improved troubleshooting to resolve data inconsistencies

- increased educational programmes targeting the different urban and rural environments to reduce the estimated undiagnosed population.

International authorities on diabetes have predicted the exponential increase in the burden of the disease to be most severe in developing countries such as SA. The DHIS could be a valuable tool to inform initiatives that are better designed for the challenges of the SA public health sector.

More quality research will enable the creation of specific and sustainable solutions that are better suited to the landscape of public healthcare in Africa.

\section{Declaration. None.}

Acknowledgements. We thank the Eastern Cape Department of Health for access to the data.

Author contributions. NS: researched the data, conducted the data analysis, generated the graphs and prepared the first draft of the manuscript; VSS: reviewed, edited and contributed to the introduction and discussion of the article.

Funding. None.

Conflicts of interest. None.

1. Pastakia SD, Pekny CR, Manyara SM, Fischer L. Diabetes in sub-Saharan Africa - from policy to practice to progress: Targeting the existing gaps for future care for diabetes. Diabetes Metab Syndr 2017;10:247. https://doi.org/10.2147/DMSO.S126314

2. International Diabetes Federation. IDF Diabetes Atlas. 8th ed. Brussels: IDF, 2017. https://www.idf. org/our-activities/advocacy-awareness/resources-and-tools/134:idf-diabetes-atlas-8th-edition.html (accessed 18 October 2019).

3. National Treasury. Estimates of national expenditure. 2018. http://www.treasury.gov.za/documents national\%20budget/2018/enebooklets/Vote\%2016\%20Health.pdf (accessed 18 October 2019).

4. Massyn N, Padarath A, Peer N, Day C. District Health Barometer 2016/17. Pretoria: Health Systems Trust, Massyn N, Padarath A, Peer N, Day C. District Health Barometer 2016/17. Pretoria: Health Systems Trust,
2018. http://www.hst.org.za/publications/Pages/District-Health-Barometer-201617.aspx (accessed 2018. http://www.
18 October 2019).

18 October 2019).
5. Mutyambizi C, Pavlova M, Chola L, Hongoro C, Groot W. Cost of diabetes mellitus in Africa: A systematic review of existing literature. Global Health 2018;14(1):3. https://doi.org/10.1186/s12992 017-0318-5

6. Motta LA, Shephard MD, Brink J, Lawson S, Rheeder P. Point-of-care testing improves diabetes management in a primary care clinic in South Africa. Prim Care Diabetes 2017;11(3):248-253. https:// doi.org/10.1016/j.pcd.2016.09.008 
7. Sahadew N, Singaram VS, Brown S. Distribution, incidence, prevalence and default of patients with diabetes mellitus accessing pablic heltheare in the 11 districts of KwaZulu-Natal, South Africa. S Afr Med J 2016;106(4):389-393. https://doi.org/10.7196/SAMJ.2016.v106i4.10143

8. Eastern Cape. 2018. https://en.wikipedia.org/wiki/Eastern_Cape (accessed 18 October 2019).

9. Pillay S, Lutge E, Aldous C. The burden of diabetes mellitus in KwaZulu-Natal's public sector: A 5-year perspective. S Afr Med J 2016;106(4):384-388. https://doi.org/10.7196/SAMJ. 2016.v106i4.9920

10. International Diabetes Federation. IDF Diabetes Atlas. 6th ed. Basel: IDF, 2013. https://www.idf.org/elibrary/epidemiology-research/diabetes-atlas.html (accessed 21 October 2019).

11. International Diabetes Federation. IDF Diabetes Atlas. 7 the ed. Basel: IDF, 2015, https://www.idf.org/elibrary/epidemiology-research/diabetes-atlas.html (accessed 21 October 2019).

library/epidemiology-research/diabetes-atlas.html (accessed 21 October 2019). South Africa. S Afr Med J 2008;98(7):549-552.

South Africa. S Afr Med 2008,98(7):549-552. Braa J, Hedberg C. The struggle for district-based health information sy

Inform Soc 2002;18(2):113-127. htps.//ai.org/10.1080/019722402 14. Matwa P, Chabeli MM, Muller M, Levit NS. Experiences and guidelines for footcare practices of patients with diabetes mellitus. Curationis 2003;26(1):11-21. https://doi.org/10.4102/curationis.

15. Williamson L, Stoops N, Heywood A. Developing a district health information system in South Africa: A social process or technical solution? Stud Health Technol Inform 2001;84:773-777. https://do org/10.3233/978-1-60750-928-8-773
16. Levitt NS, Katzenellenbogen JM, Bradshaw D, Hoffman MN, Bonnici F. The prevalence and identification of risk factors for NIDDM in urban Africans in Cape Town, South Africa. Diabetes Care 1993:16(4):601-607. https://doi.org/10.2337/diacare.16.4.601

17. Massey CN, Appel SJ, Buchanan KL, Cherrington AL. Improving diabetes care in rural communities: An overview of current initiatives and a call for renewed efforts. Clin Diabetes 2010;28(1):20-27. https://doi.org/10.2337/diaclin.28.1.20

18. Amod A, Berg G. The 2012 SEMDSA guidelines for the management of type 2 diabetes. J Endocrinol Metab Diabetes S Afr 2012;17(2):S4. http://www.cdediabetes.co.za/uploads/images/files/SEMDSA\%20 Guideline \%20for\%20the\%20Management\%20of\%20Type\%202\%20Diabetes_2012.pdf (accessed 18 October 2019)

19. Shaw V. Health information system reform in South Africa: Developing an essential data set. Bull World Health Organ 2005;83(8):632-636

Accepted 22 May 2019. 